\title{
THERMAL STIFFNESS - A KEY ACCURACY INDICATOR OF MACHINE TOOLS
}

\author{
A. P. Kuznetsov ${ }^{1}$, H. J. Koriath ${ }^{2 *}$ \\ ${ }^{1}$ Moscow State University of Technology STANKIN, Department of Machine Tools, Moscow, Russia \\ ${ }^{2}$ Fraunhofer Institute for Machine Tools and Forming Technology IWU, Automation and Monitoring, Chemnitz, \\ Germany \\ *Corresponding author; e-mail: koriath@iwu.fraunhofer.de
}

\begin{abstract}
Static and dynamic stiffness [ $\mathrm{N} / \mathrm{m}$ ] determine the ability of solids to resist constant and variable loads. Both elastic characteristics of a machine tool effect their quality assessment. Thermal stiffness (comprising heat stiffness and temperature stiffness) $[\mathrm{W} / \mu \mathrm{m}]$ is a key accuracy indicator of the machine tool's ability to resist temperature influences. The proposed method creates the thermo-physical structure of a machine tool, based on a set of homogeneous heat-active elements and quasi-thermostable links. Quasi-thermostable links retain constant properties when the thermal state of the heat-active elements changes within a given range, building and determining their spatial and temporal relative position. The structural formula is given: $<$ S-thermal link $>-<F$-function of the thermal behavior of a heat-active element $>-<$ S-thermal link $>$. When exposed to heat, heat-active elements change their temperature and thermoelastic properties change their temperature and thermoelastic properties with stress, strain, distortion. Thermal behavior F-functions characterize these changes over time. Thermal energy causes a heat exchange in the machine tool and leads to temperature differences, thermoelastic stresses and geometrical deformations. The material used in machine tools enables the thermal conduction, convection and radiation due to its dimensions, volume and surface area, thermal conductivity. Elasticity effects base on thermal linear expansion coefficient, modulus of elasticity, thermal energy storage due to its heat capacity. The analysis of the structural formula defines and describes generalized thermal stiffness indicators of a machine tool as a reaction to thermal effects when the heat sources are constantly active and when the heat source is absent, but only the ambient temperature changes. This paper presents relationships between the thermal stiffness and the thermo-physical property indicators of the machine tool. Examples of thermal stiffness are described for several machine tool types.
\end{abstract}

Keywords:

Machine tool; thermo-physical model; thermoelasticity, thermal stiffness; accuracy indicator

\section{INTRODUCTION}

Since 1950, scientists have carried out numerous research investigations on thermal processes and effects occurring in machine tools during their operation, including the following areas:

1) definition of values for temperatures and thermal deformations in machine tools of different types; [Kuz 2018]

2) determining the influence of heat sources and thermophysical parameters on the thermal mode of the machine tool, its parts, and assemblies; [Kuz 2019]

3) investigating the impact of design features of separate units and machine parts on their temperature fields and deformations. [Mek 2009]

4) determining the influence of the thermal mode of the machine on the parameters of machining accuracy and geometrical error of individual units and machine parts; [May 2012]

5) numerical methods (mainly based on the finite element method) to determine and evaluate temperatures and thermal deformations of parts, assemblies, machine tools; [Yto 2010]

6) investigation of means, methods, and techniques of compensation, numerical compensation, reduction of temperature deformations and temperature fields, control of temperature fields and deformations of machine tools, their units and parts; [Gro 2015]

7) research on possible applications of new kinds of materials for improving thermal stiffness and thermal stability of machine tools.

During the past 30 years, the International Organization for Standardization (ISO) has developed several standards for test procedures on geometrical accuracy under static or no load conditions: ISO 230-3 (Determination of Thermal Effects); ISO 10791-10 (Thermal Displacement in Machining Centers); ISO 13041-8 (Thermal Displacement in Lathes), ISO 16907-2015 (Numerical Compensation of Geometric Errors), which define test methods for evaluating thermal influences that lead to thermal deformation of a machine tool structure and/or its positioning systems. 
Table 1 Thermo-physical transformations

\begin{tabular}{|l|l|l|l|l|l|l|}
\hline $\begin{array}{l}\text { thermo-physical } \\
\text { transformation }\end{array}$ & geometry & power & heat flow & temperature & $\begin{array}{l}\text { thermo- } \\
\text { elasticity }\end{array}$ & deformation \\
\hline properties & $\begin{array}{l}\text { volume, } \\
\text { mass, } \\
\text { density, } \\
\text { surface }\end{array}$ & $\begin{array}{l}\text { energy } \\
\text { efficiency, } \\
\text { heat } \\
\text { generation }\end{array}$ & $\begin{array}{l}\text { conduction, } \\
\text { convection, } \\
\text { radiation }\end{array}$ & $\begin{array}{l}\text { thermal } \\
\text { resistance }\end{array}$ & $\begin{array}{l}\text { Young's } \\
\text { modulus, } \\
\text { stress }\end{array}$ & $\begin{array}{l}\text { elongation, } \\
\text { bending, } \\
\text { strain }\end{array}$ \\
\hline metrics & $\begin{array}{l}\boldsymbol{k}[\mathrm{N} / \mathrm{m}] \\
\text { stiffness } \\
\text { coefficient }\end{array}$ & $\begin{array}{l}\mathbf{Q}[\mathrm{J}] \\
\text { amount of heat }\end{array}$ & $\begin{array}{l}\boldsymbol{K}_{\boldsymbol{\theta} j}[\mathrm{~W} / \mathrm{K}] \\
\text { heat } \\
\text { elasticity, } \\
\boldsymbol{K}_{\boldsymbol{\alpha} \boldsymbol{j}}[\mathrm{K} / \mathrm{W}] \\
\text { heat stiffness }\end{array}$ & $\begin{array}{l}\boldsymbol{\Delta}[\mathrm{K}] \\
\text { temperature } \\
\text { difference } \\
\mathbf{C}[\mathrm{J} / \mathrm{K}] \\
\text { heat } \\
\text { capacity }\end{array}$ & $\begin{array}{l}\boldsymbol{\beta} \text { thermal } \\
\text { linear } \\
\text { elongation, } \\
\boldsymbol{K}_{\boldsymbol{T} j}[\mathrm{~K} / \mu \mathrm{m}] \\
\text { temperature } \\
\text { stiffness }\end{array}$ & $\begin{array}{l}\boldsymbol{K}_{\boldsymbol{Q} \boldsymbol{j}}=K_{T j} K_{\theta j} \\
{[\mathrm{~W} / \mu \mathrm{m}]} \\
\text { thermal } \\
\text { stiffness }\end{array}$ \\
\hline
\end{tabular}

Machine tool errors are typically measured values that provide an objective assessment of machine tool properties, including the machine tool's ability to withstand constant, variable, and periodic force and thermal influences.

\section{DEFINITION OF STIFFNESS}

\subsection{Mechanical stiffness}

Thus, the relationship describes the elastic displacements of machine units and parts by

$F=k \cdot x$

that links the stiffness coefficient $k$ as static force $F /$ displacement $x$ or dynamic force / velocity $\dot{x}$. The dynamic behavior (amplitude and frequency) of the elastic system is described by the equation

$m \ddot{x}(t)+c \dot{x}(t)+k x(t)=F(t) ;$

from which the dynamic stiffness coefficient $k$ is determined. Therefore, the machine tool's static and dynamic characteristics are specified by the dependencies that express the relation of the output variable of the system $x$ - the displacement or the speed of this displacement $\dot{x}$ to the input variable $F$ - the force.

\subsection{Thermal stiffness}

A thermo-physical transformation consists of heat flow through conduction, convection, and radiation causing temperature changes and thermoelastic stress, resulting in strain and deformations, see Table 1. Thermal conduction follows the heat equation for rigid bodies and mechanical contact: $Q=\frac{\lambda A t \Delta T}{L}[\mathrm{~J}]$. Thermal convection follows the heat equation: $Q=\alpha A t \Delta T[\mathrm{~J}]$. Thermal radiation follows the absorption equation (reflection $\rho$, absorption $\alpha$, transmission $\tau$ ): $\Phi=\alpha \Phi_{s}$ [W]. Heat exchange between thermal capacities of two bodies follows: $c_{1} m_{1} \cdot\left(T_{1}-T_{m}\right)=$ $c_{2} m_{2} \cdot\left(T_{m}-T_{2}\right)$.

The thermoelastic displacement of the machine parts is calculated by the linked equations for the thermal conductance and thermoelasticity:

$\nabla U_{i}+\frac{1}{1-2 \mu} \cdot \frac{\partial e}{\partial i}-\frac{2 \beta(1+\mu)}{(1-2 \mu)} \cdot \frac{\partial T}{\partial i}=0 ;$

where $\mathrm{i}=\mathrm{x}, \mathrm{y}, \mathrm{z} ; \nabla=\frac{\partial^{2}}{\partial \mathrm{x}^{2}}+\frac{\partial^{2}}{\partial \mathrm{y}^{2}}+\frac{\partial^{2}}{\partial \mathrm{z}^{2}} ;-$ Laplace operator; $\mu-$ Poisson coefficient; $e=\frac{1-2 \mu}{2 G \cdot(1+\mu)} \cdot\left(\sigma_{x x}+\sigma_{y y}+\sigma_{z z}\right)+3 \beta T$; - volumetric expansion ratio; $\beta$ - thermal linear elongation coefficient; $G=\frac{E}{2(1+\mu)}$; - shear module; $E$ - Young's modulus of elasticity; $\left(\sigma_{x x} ; \sigma_{y y} ; \sigma_{z z}\right)$ - nominal stress; $T$ temperature distribution.
This leads to a prominent and essential difference in the application of stiffness and thermal stiffness, since, in the case of static and dynamic stiffness, the place and direction of force application and the place of their measurement are fixed. In the case of the machine tool's thermal stiffness, the place of measurement is fixed (e. g. TCP), but the size and location of the position and direction of the thermal impact are not clearly defined. Such an "objective" assessment, only based on the equation's solution of the related problem of thermal elasticity and heat conductivity, cannot provide a basis for assessing the value of the machine tool's thermal stiffness and, therefore, nor control the thermal behavior of the machine tool at all stages of its life cycle.

The most desirable characteristic is an indicator that most clearly defines this property of the machine tool. The concept of stiffness is known as the ability to resist the force influences with elastic deformations, which has the dimension $[\mathrm{N} / \mu \mathrm{m}]$, and a test method. For thermal deformations, there are similar attempts to define the concept of "thermal stiffness" as the ability of the structure to resist heat influences with elastic deformations and determine it as a ratio of power losses at a certain speed and the resulting maximum deformation in the selected coordinates.

\section{THERMO-PHYSICAL STRUCTURE}

Thus, there is a need for a deeper and more comprehensive analysis of the mechanism of formation and change of thermal processes in machine tools concerning the definition and justification of the machine tool's evaluation and concept of thermal stiffness.

\subsection{Elements and links}

The thermo-physical structure of a machine tool can be described as an interconnected set of homogeneous heatactive elements forming and determining the tool's and the workpiece's relative position in space and time. The heatactive element is a part (or unit) that changes its thermoelastic properties, or it transmits the thermoelastic effect. This leads to a change in the initial state of the relative position of the tool and workpiece in space and time. Heat-active elements form a thermo-physical structure with the support of quasi-thermostable links that retain their properties when the heat state of the heat-active elements changes within the specified range. Consequently, the combination of heat-active elements and their links forms the thermo-physical structure in the machine tool's space. Due to the heat influence over time, the heat-active elements change their thermoelastic properties. These changes over time are characterized by the function of the heat-active elements' thermal behavior. The combination of elements by their links and their thermal behavior's function forms the thermo-physical structure of 
the machine tool in space and time. Thus, a new view on the thermal system of the machine tool has led to a definition of new concepts and new terms.

Heat-active elements in a machine tool comprise spindle, ball screw, column, bed, table, spindle headstock, etc. As a rule, these elements have relatively large linear dimensions, heat dissipation area, weight, and are part of a kinematic chain that determines the relative position of the tool and workpiece. Other components of the machine too have small linear sizes and low temperature, small weight, and consequently also low heat capacity, small area of a heat-dissipating surface, so that they cannot essentially influence the machine tool's thermal condition.

Quasi-thermostable links are defined by assembly interfaces between elements (guides, bearings, nut, etc.), and by locations of their thermal symmetry or gravity center of masses. For example, assembly interfaces include the fixation points of the spindle, ball screw, motion transmission points, and points that restrict (or exclude) the movement of elements, etc. Consequently, the position of the spindle support does not remain constant when the spindle is heated, but the support ensures a relatively stable relative position of the spindle and spindle headstock when heated. Another link is the interface between the table, spindle headstock, and ball screw carried out via the screw nut's transmission, which also ensures a relatively stable relative position when heated. This is the reason why these links are called quasi-thermostable, as they only remain the most stable under thermal conditions and maintain their initial relative position to any other parts, unit, or component of the machine tool. The analysis of links and interfaces of heat-active elements in machine tools allows classifying them according to the number of link conditions (restrictions) imposed by the linked elements on the displacements relative to each other. The coordinates of surface points ought to satisfy the interface equations, which this research determines as the equations of quasithermostable links.

\subsection{Thermoelastic structure}

In order to design a thermoelastic structure of a machine tool, it is necessary to define in detail the types of typical quasi-thermostable links, heat-active elements, and functions of thermal behavior.

It is useful for a heat-active element to choose a beam of any cross-section which movement is limited by quasistable links applied on its border. In general, a beam end can move in three orthogonal axes and elastically rotate around them, if no link restricts these linear and rotational motions. The simplest heat-active element with one rigid link is an immovable beam at one end, and the second end can move freely in the axial directions. Regardless of the number of heat-active elements, all coordinate structures will have the form of a closed polygon, but the desired one will be a closing vector that connects the first and last quasithermostable link. Any of the thermoelastic structures constructed in this way can be transformed into three independent structures - its projections to the coordinate planes XOY, XOZ, ZOY. For each such projection, a mathematical expression can be formulated for determining the closing vector. The analysis of this expression will make it possible to find the value of thermal stiffness and heat resistance in the selected directions and planes, and also estimate the influence of individual structural components.

Figure 1 shows typical coordinate thermoelastic structures with varying degrees of complexity. Thus, a coordinate thermoelastic structure can consist of one, two, three, or more elements.

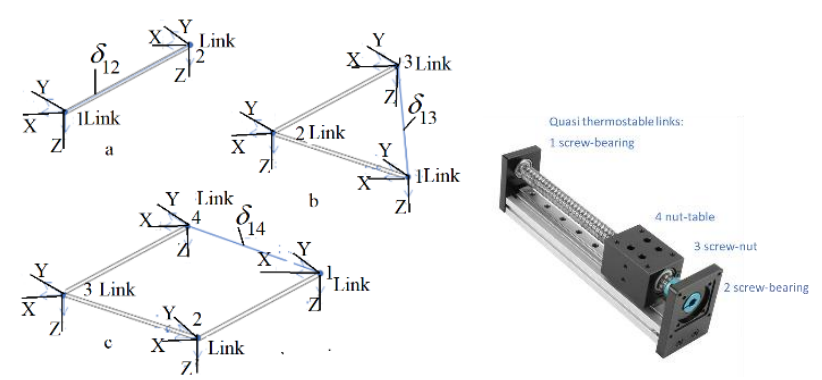

Fig. 1: Three coordinate thermoelastic structures: a - oneelement, $b$-two-elements, $c$ - three-elements, $d$ - quasi thermostable links in a ball-screw-nut-table unit.

Then the simplest thermoelastic structure will consist of a quasi-thermostable link and a heat-active element characterized by the function of thermal behavior and a quasi-thermostable link. If a heat-active element transmits a thermoelastic effect through an intermediate link, such a thermoelastic structure consists of three links and functions of thermal behavior. An example of such a structure is the thermoelastic displacement of a table caused by the heating of a ball screw. In this case, the quasi-thermostable links are the ball screw bearings and the screw-nut transmission. Another type of heat-active element is a plate or disk of any cross-section, where their motion and deformation is limited by quasi-stable links applied on its border. A typical element with a single rigid link can often be considered a column of a metal cutting machine, which is immovably fixed to the bed. The bed itself is an example of a heat-active element, with no links limiting motion and deformations.

\subsection{Temperature fields}

Non-uniformity and inhomogeneity of the temperature field of heat-active elements lead to temperature stresses and deformations, which is an essential factor in their thermal behavior and causes linear and angular changes in the heat-active elements. It is known as the N.A. Yaryshev ratio that estimates $\zeta$ - the inhomogeneity of the temperature field:

$\zeta=\frac{K n}{B i_{v}}=\frac{1}{\sqrt{B i_{v}^{2}+1,437 B i_{v}+1}}$

If the distribution of the temperature field is homogenous, then the following applies: $\zeta=1 ; \mathrm{B} i_{v} \rightarrow 0$. The higher the inhomogeneity of the temperature field, the lower the value $\zeta=0 ; B i_{v} \rightarrow \infty$ N.A. Yaryshev's ratio. Consequently, the Biot number characterizes the inhomogeneity degree of the temperature field. Thus, if the Biot number for practical application is less than 0.1 , the temperature field is close to homogenous, and in this case there will be no bending of the plate. If the Biot number is greater than 0.1 , the temperature field is inhomogeneous, which will cause bending temperature deformations. The Biot number $B i=$ $\frac{\alpha L}{\lambda}$ depends on and is determined by $\lambda$ heat conductivity $[\mathrm{W} / \mathrm{mK}]$ and the size $L[\mathrm{~m}]$.

For machine tools, $\alpha$ values at free convection vary between 4 and 15 , and $\lambda$ values for relevant steels vary between 40 and 50 , and $\lambda$ values for cast iron range from 50 to 65 . Then, the values of the Biot number ranges can be determined: for steel parts $B i=(0.08-0.375)$, for cast iron parts $B i=(0.062-0.3)$. Therefore, the $L$ size of heatactive elements, for which the temperature field can be considered relatively homogeneous, will be within the following ranges: for steels - less than 1.13-0.27 m, for cast iron - less than 1.61-0.33 m. 


\section{THERMOELASTIC DEFORMATIONS}

\subsection{Kinematic chain}

Therefore, a thermo-physical model of a machine tool can most often be built in three ways: based on heat-active elements from only beams, based on heat-active elements from plates or disks, or based on a combination of plate and beam-shaped heat-active elements.

Quasi-thermostable links, depending on the restrictions they create in each coordinate direction of the element's motion, can be rigid, elastic, with a gap, movable, allowing rotation, or representing their combinations.

An example of a rigid link may be the fixation of a column to the machine bed. Elastic links are typical for the fixation points in the ball screw and spindle supports. Sometimes, one of the elastic links can be taken as a rigid one if their stiffness ratio differs significantly from each other. The condition under which one of the elastic supports is considered rigid can be found in the following ratio:

$\frac{\delta_{1}}{\delta_{2}}=\frac{k_{2}}{k_{1}}$;

where $\delta_{1} ; \delta_{2}$ - thermoelastic deformations at bearing 1 and $2 ; k_{1} ; k_{2}-$ stiffness of bearing 1 and 2 .

If the ratio $\frac{k_{2}}{k_{1}}$ is close to zero, there is practically no displacement of the first bearing, and it can be considered a rigid link of this bearing with the heat-active element. All links between two heat-active elements of the machine too are also subject to the conditions of interchange and compatibility of linear and angular motions, i.e. each heatactive element of the machine tool performs only those displacements that are allowed by their mutual links between each other.

When building coordinate systems in positions of quasithermostable links, thermoelastic structures of machine elements can be obtained in space. These thermoelastic structures of machine tools equivalently replace the machine tool's construction when considering the thermoelastic displacements in the machine tool caused by the heating of its parts and assemblies. Thus, if the machine tool elements are units and parts that determine its kinematic chain, such a structure will characterize the thermoelastic structure of the machine tool's kinematic chain and determine the nature of thermal processes due to this method and the location of heat sources. The analysis of such thermoelastic structures will make it possible to evaluate the machine's kinematic chain regarding restrictions of thermal displacements. Moreover, the process of such an analysis is essential in a structuralkinematic thermophysical analysis, assessing the thermal stiffness of the entire machine tool.

\subsection{Function of thermal behavior}

The generalized structural formula of the machine tool (see Fig.2) has the following form:

$<$ quasi-thermostable link $C_{i}>\quad-<$ function of thermal behavior $F_{i}>$ - <quasi-thermostable link $C_{i}>$

and is equal to the number of heat-active elements.

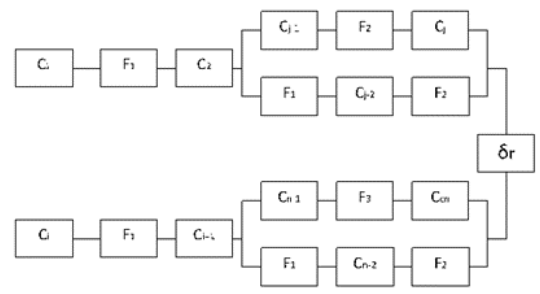

Fig. 2: General thermo-physical structural scheme.
Figure 3 presents examples of thermoelastic kinematic structures of machine tools, including rigid quasithermostable links.
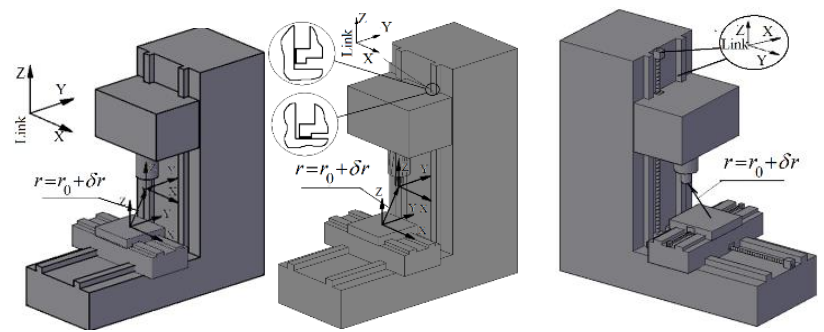

Fig. 3: Examples of thermoelastic kinematic structures of machine tools with rigid vertical columns.

In general, the thermoelastic structure of any machine tool consists of $i$ heat-active element $i=(n-1)$, in places of quasi-thermostable links $n$ of which $n$ coordinate systems are built. The position of one coordinate system relative to another is determined by the radius vector $r_{i}$ and the matrix $M_{i}$ of their mutual position. In a sequential transition from one coordinate system to another, the position $\delta r$ of a fixed point (which is set in the coordinate system of the tool) is determined in the coordinate system of the part or vice versa. The same relative displacement $\delta r$ of the tool and the workpiece can also be defined as the difference between the sum of the two vectors having the same origin with different end points, respectively in the position of the tool point and the workpiece. The results of such calculations will be equal and will determine the error (difference) of the mutual change of vector positions in the adopted coordinate system.

\subsection{Thermal displacement}

During heating of the machine tool, its heat-active elements change the linear sizes on value $\delta r_{i}^{0}$, and inhomogeneity of a temperature field causes their angular rotation (bending) defined by a matrix of angular temperature deformations $\delta \alpha_{i}$ of heat-active elements of the machine tool

$\boldsymbol{B}_{i}^{\alpha}=\boldsymbol{B}_{i x}^{\alpha} \times \boldsymbol{B}_{i y}^{\alpha} \times \boldsymbol{B}_{i z}^{\alpha} ;$

see Tab. 2, and depending on the machine tool and workpiece accuracy, the $2^{\text {nd }}$ order matrices $\left(\delta \alpha_{x}^{2}, \delta \alpha_{x}, \delta \alpha_{y}\right)$ and higher order $\left(\delta \alpha_{z} \delta \alpha_{x} \delta \alpha_{y} ; \delta \alpha_{y} \delta \alpha_{x}^{2} ; \delta \alpha_{y}^{2} \delta \alpha_{z}^{2}\right)$ are assumed as zero. The value of the relative displacement $\delta r$ of the tool and workpiece due to the heating of the machine tool's thermoelastic elements will be determined by the difference between the values of the machine tool's thermoelastic system, respectively, after and before heating. Then the general expression for estimating the value and nature of temperature deformations built by the thermoelastic deformation of the machine tool, the following equation will be applied:

$$
\begin{aligned}
& \delta r=\sum_{i=1}^{n-1}\left(\prod_{i=1}^{n-(i+1)} M_{i}\right) \times K_{i} \times \delta r_{i}^{0}+\sum_{i=1}^{n-1}\left(\prod_{i=1}^{n-(i+1)} M_{i}\right) \times \\
& \left(\boldsymbol{B}_{i}^{\alpha}\right)^{I} \times r_{i}+\sum_{i=1}^{n-1}\left(\prod_{i=1}^{n-(i+1)} M_{i}\right) \times\left(\boldsymbol{B}_{i}^{\alpha}\right)^{I} \times K_{i} \delta \times r_{i}^{0} ;
\end{aligned}
$$

where $\delta r=\left|\delta r_{x} \delta r_{y} \delta r_{z} 1\right|^{T}$ - error matrix of mutual relative position of the tool and the part, caused by thermoelastic deformations of the machine; $\delta r_{i}^{0}=\left|\delta r_{i x}^{0} \delta r_{i y}^{0} \delta r_{i z}^{0} 1\right|^{T}$ - matrix of thermoelastic deformations of heat-active elements at quasi-thermostable links; $K_{i}$ - relative stiffness coefficient of a heat-active element at quasi-thermostable links;

$\left(\boldsymbol{B}_{i}^{\alpha}\right)^{I}=\boldsymbol{B}_{i}^{\alpha}-\boldsymbol{I}$;

where I - unity matrix.

For describing the method mentioned above, a typical 


$$
=\left|\begin{array}{cccc}
\left(1-\frac{\delta \alpha_{y}^{2}}{2}\right)\left(1-\frac{\delta \alpha_{z}^{2}}{2}\right) & -\left(1-\frac{\delta \alpha_{y}^{2}}{2}\right) \delta \alpha_{z} & -\delta \alpha_{y} & 0 \\
-\delta \alpha_{x} \delta \alpha_{y}\left(1-\frac{\delta \alpha_{z}^{2}}{2}\right)+\left(1-\frac{\delta \alpha_{x}^{2}}{2}\right) \delta \alpha_{z} & -\delta \alpha_{z} \delta \alpha_{x} \delta \alpha_{y}+\left(1-\frac{\delta \alpha_{y}^{2}}{2}\right)\left(1-\frac{\delta \alpha_{z}^{2}}{2}\right) & -\delta \alpha_{x}\left(1-\frac{\delta \alpha_{y}^{2}}{2}\right) & 0 \\
\delta \alpha_{y}\left(1-\frac{\delta \alpha_{x}^{2}}{2}\right)\left(1-\frac{\delta \alpha_{z}^{2}}{2}\right)+\delta \alpha_{x} \delta \alpha_{z} & -\delta \alpha_{y} \delta \alpha_{z}\left(1-\frac{\delta \alpha_{x}^{2}}{2}\right)+\delta \alpha_{x}\left(1-\frac{\delta \alpha_{z}^{2}}{2}\right) & \left(1-\frac{\delta \alpha_{x}^{2}}{2}\right)\left(1-\frac{\delta \alpha_{y}^{2}}{2}\right) & 0 \\
0 & 0 & 0 & { }_{1}
\end{array}\right|
$$

Table 2: Matrix of angular temperature deformation.

example of a heat-active element is considered, such as a spindle, or a ball screw, or long parts of any cross-section, reduced to a beam. Displacement of a thermostable link $\delta r_{i}^{0}=\mathrm{U}$ during heating will be defined by the decision of the connected one-dimensional problem of thermoelasticity, with restrictions of an arrangement of bearings as rigid thermostable links, and a problem of thermal conduction with thermal loads - heat emission in bearing supports. Then the solution of the equation system is:

$\frac{\partial^{2} U}{\partial x^{2}}=\beta \cdot \frac{\partial T}{\partial x} ;$

with boundary conditions $\varepsilon_{x x} \cdot E-\beta \cdot E \cdot T=\sigma_{x x}$ for $x=0$; and $\varepsilon_{x x} \cdot E-\beta \cdot E \cdot T=-\sigma_{x x}$ for $x=l$.

Considering the specified stiffness $k_{1} ; k_{2}$ (front and rear spindle bearings), and considering force $F=k \cdot U$ and $\sigma_{x x}=\frac{F}{A}=\frac{k \cdot U}{A} ; \varepsilon_{x x}=\frac{\partial U}{\partial x}$ the cross-sectional area of the beam results in the following equation:

$\frac{U(l, r)}{\int_{0}^{l} \beta T(x, t) d x}=\frac{k_{\Sigma}}{k_{2}}-\frac{k_{\Sigma}}{k_{2}} \cdot \frac{l F_{p r}}{E F \int_{0}^{l} \beta T(x, t) d x}+\frac{k_{\Sigma}\left(k_{1}^{-1}+\frac{l}{E F}\right) \delta_{0}}{\int_{0}^{l} \beta T(x, t) d x}=\frac{k_{\Sigma}}{k_{2}}-$
$\frac{k_{\Sigma} k_{p r}}{k_{2} k_{e}}+k_{\Sigma}\left(k_{1}^{-1}+k_{e}^{-1}\right) k_{0} ;$

or for $n=2$ with $x=l$ the resulting equation is

$U(l, r)=\beta \cdot K_{i} \int_{0}^{l} T(x, t) d x=\beta \cdot K_{i} \cdot l \cdot T_{\varnothing}(x, t) ;$

where $K_{i}=\left[\frac{k_{\Sigma}}{k_{2}}\left(1-\frac{k_{\mathrm{pr}}}{k_{b}}\right)+K_{0}\left(1-\frac{k_{\Sigma}}{k_{2}}\right)\right]$ - relative stiffness coefficient of a heat-active element at quasi-thermostable links; $k_{\Sigma}=\left(k_{1}^{-1}+k_{e}^{-1}+\frac{l}{E F}\right)^{-1}$ - total stiffness; $k_{b}=\frac{E F}{l}-$ mechanical stiffness of the element; $F_{p r}$ - preload (tension, compression) force; $k_{p r}=\frac{F_{p r}}{\int_{0}^{l} \beta T(x, t) d x}-$ preload stiffness; $k_{0}=\frac{\delta_{0}}{\int_{0}^{l} \beta T(x, t) d x}-$ relative stiffness coefficient of clearance; $\delta_{0}$ - clearance of the thermostable link.

\subsection{Distribution of temperatures}

To determine the distribution of temperatures $T(x)$ and/or the average $T_{\varnothing}(x)$ of a heat-active element, e.g. spindle or ball screw, the thermal model is assumed as a cylinder (or beam of any cross-section) of finite length. The heat exchange takes place on the cylinder surfaces with the environment with a heat convection coefficient $\alpha$. The heat is transferred through the surfaces at the bearing fixing points. The lengths of the heat-active element and the real lengths of the same element can be used to determine the thermal model's diameter from the equality of the Biot number or Fourier criteria:

$d_{m p}=\frac{\alpha_{\phi}\left(\sum d_{i}^{2} l_{i}-\sum d_{i}^{2} l_{j}\right)}{\sum \alpha_{i} d_{i}^{2} l_{i}-\sum \alpha_{j} d_{i}^{2} l_{j}+\sum \alpha_{n} d_{i}^{2}\left(d_{i}^{2}-d_{j}^{2}\right)} ;$

where $i, j-$ is the number of spindle cross-sections of the same external or internal diameter; $d_{i}, d_{j}$ - is the value of the external and internal diameter respectively; $l_{i}, l_{j}-$ is the length of the cross-section with the same external or internal diameter; $\alpha_{\emptyset}$ is the accepted heat convection coefficient of the heat model or calculated from the relationship:

$\alpha_{\emptyset}=\sum \alpha_{i} A_{i}+\frac{\sum \alpha_{n} A_{n}}{\sum A_{i}+\sum A_{n}} ;$

where $\alpha_{i}$ is the heat convection coefficient of the heat transfer surface $A_{i}$ (internal and external), $\alpha_{t i}$ is the heat convection coefficient of the beam end surfaces. Then the solution of the equation regarding the temperature distribution with the specified boundary conditions will be determined as follows, see fig. 4 :

$T_{1}(x)=\frac{Q_{2}}{A m_{2} \lambda} \cdot \frac{D_{m}}{1+\frac{m_{1}}{m_{2}} \cdot D_{m} \tanh \left(m_{1} L_{1}\right)} \cdot \frac{\cosh \left(m_{1} x\right)}{\cosh \left(m_{1} L_{1}\right)}+\frac{Q_{1}}{A m_{1} \lambda}$.

$\frac{\cosh \left(m_{1} x\right)}{\cosh \left(m_{1} L_{1}\right)} \cdot \frac{\left[\cosh \left(m_{1} L_{1}\right)-\sinh \left(m_{1} L_{1}\right)\right]\left(\frac{m_{1}}{m_{2}} D_{m}-1\right)}{1+\frac{m_{1}}{m_{2}} \cdot D_{m} \tanh \left(m_{1} L_{1}\right)}+\frac{Q_{1}}{A m_{1} \lambda}$.

$\left[\cosh \left(m_{1} x\right)-\sinh \left(m_{1} x\right)\right]$;

where $D_{m}=\frac{K_{m} e^{2\left(L-L_{1}\right) m_{2}+1}}{K_{m} e^{2\left(L-L_{1}\right) m_{2}-1}} ; K_{m}=\frac{m_{2}+\left(\frac{\alpha_{m}}{\lambda}\right)}{m_{2}-\left(\frac{\alpha_{m}}{\lambda}\right)} ; m_{1}^{2}=4 \frac{\alpha_{1}}{\lambda d} ; m_{2}^{2}=$ $4 \frac{\alpha_{2}}{\lambda d}$ or $m_{1,2}^{2}=\frac{P \alpha_{1,2}}{\lambda \cdot A}$; where $\mathrm{P}-$ perimeter, $A$ - square area.

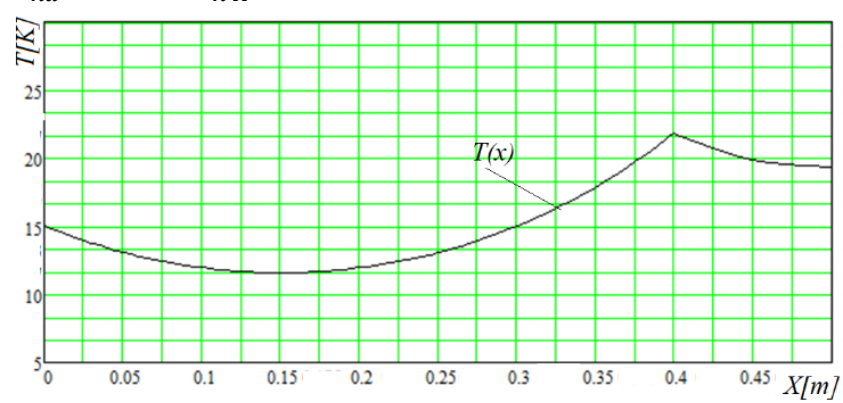

Fig. 4: Temperature $T(x)$ distribution over a beam length $L$ of a heat-active element.

\subsection{Thermal machine model}

The methodology of the machine tool's temperature model is also based on the above-described methodology of the machine tool's thermal physical structure. The structural components have the same description form, however, in contrast to the structural components, which are subject to thermoelastic deformations; they are described by the following equation:

$<S$ - thermal link $>-<F-$ function of thermal behaviour of a heat-active element $>-<S$ - thermal link $>$.

The general temperature model of a machine tool and its structure can be represented as a system of $n$ bodies (heatactive elements) with heat sources and flows (thermal links) located in space. Parts and units of a machine tool exchange thermal energy with the environment and among themselves. It is necessary to find the spatial and temporal temperature distribution of parts and units (bodies - heatactive elements) for different operation modes. Moreover, the thermophysical properties of materials, heat conductivity between the bodies, and the power of sources and energy flows do not depend on temperature. The 
mathematical model of such a system of bodies [Kuz 2019] with heat sources consists of $n$ linear equations of therma conductance:

$\nabla^{2} T_{k}\left(x_{i}, t\right)+\frac{Q_{e k}\left(x_{i}, t\right)}{\lambda_{k}}=\frac{1}{a_{k}} \cdot \frac{\partial T_{k}\left(x_{i}, t\right)}{t} ;$

where $x_{i}=x, y, z$ - brief coordinate record; $k=$ $1,2,3, \ldots l, \ldots n$ - body numbers in the system; $Q_{e k}=Q_{o k}+$ $Q_{i k}$ - general spatial and temporal distribution of energy sources in $k$ body; $Q_{o k}$ - amount of heat supplied to $k$ body from outside; $Q_{i k}$ - amount of heat released in body; $\lambda_{k}$, effective or true heat conductivity and $a_{k}$ - heat convection coefficient of $k$ body.

The heat flux supplied to the heat-active element from the sources is generally discharged into the environment and to other heat-active elements of the machine, and is also used to heat the element itself. Based on the energy conservation law, the heat flux $Q_{T k}$ supplied by the heat conduction from inside the $k$ body to its surface area $A_{k}$ is transferred by $Q_{\lambda k}$ to the surrounding bodies and the environment $Q_{\alpha k}$ :

$Q_{T k}=Q_{\lambda k}+Q_{\alpha k}$

Based on the Fourier law, the heat flux is brought to the element of the surface area $d A_{k}$ of the body $k$, and the whole surface $A_{k}$ is found by summing up all the elementary flows

$Q_{T k}=-\int_{A_{k}} \lambda_{k} \cdot \frac{\partial T_{k}\left(x_{i}, t\right)}{\partial n} d A_{k} ;$

Thermal flux $Q_{\lambda k l}$ from body $k$ to body $l$ will be determined on the basis of the equation

$Q_{\lambda k l}=\psi_{k l} \cdot\left[\left(T_{k}\right)_{A_{l}}-\left(T_{l}\right)_{A_{k}}\right]$;

where $\psi_{k l}$ - thermal conductance, $[\mathrm{W} / \mathrm{K}]$ - the inverse value is $R$ - thermal resistance $\psi_{k l}=\frac{1}{R_{k l}}=R_{k l}^{-1}$ between the bodies $k$ and $l ;\left(T_{k}\right)_{A_{l}}$ and $\left(T_{l}\right)_{A_{k}}$ - average surface overheating of those parts of the surface $A_{l}$ and $A_{k}$ of the bodies $k$ and $l$, which participate in the mutual heat exchange.

Summarizing all heat flows of the body $k$ to all bodies $l=$ $1,2,3, \ldots \mathrm{n}$, results in:

$Q_{\lambda k}=\sum_{\substack{l=1 \\ k \neq l}}^{n} Q_{\lambda k l}=\sum_{\substack{l=1 \\ k \neq l}}^{n} \psi_{k l} \cdot\left[\left(T_{k}\right)_{A_{l}}-\left(T_{l}\right)_{A_{k}}\right]$

Thermal flux $Q_{\alpha k}$ from the body $k$ into the environment based on the Newton-Roman law:

$Q_{\alpha k}=\psi_{\alpha k} T_{\alpha k}=\psi_{\alpha k} \cdot\left[\left(T_{k}\right)_{A_{\alpha}}-T_{\text {air }}\right]$;

where $\left(T_{k}\right)_{A_{\alpha}}$ - respectively the $k$ body temperature, from the surface of which $A_{l} \neq A_{\alpha}$ heat is exchanged with the environment with a temperature of $T_{\text {air }}$. Substituting the expressions (17), (19) and (20) in (16) results in:

$-\int_{A_{k}} \lambda_{k} \frac{\partial T_{k}\left(x_{i}, t\right)}{\partial n} d A_{k}=\psi_{\alpha k} T_{\alpha k}+\sum_{\substack{l=1 \\ k \neq l}}^{n} \psi_{k l} \cdot\left[\left(T_{k}\right)_{A_{l}}-\left(T_{l}\right)_{A_{k}}\right]$;

and assuming that the initial temperature distribution in body $k$ is $T_{k}\left(x_{i}, t\right)=T_{k}^{b}\left(x_{i}\right)$ at $t=0$.

The system of equations (16) - (21) is a general mathematical model of the temperature mode of $n$ bodies obtained with one limitation: the linear problem statement is considered, i.e., it is assumed that the physical properties of bodies $\lambda_{k}$ heat conductivity, $a_{k}$ heat convection coefficient, $\psi_{k l}$ thermal conductance and the power of sources $Q_{k}$ do not depend on temperature and that individual bodies (machine parts) are isotropic. In practice, it is not always necessary to have such detailed information about the temperature mode of the machine parts and units (bodies); sometimes, it is enough to limit the average temperature values. For this case, the system of equations (15) - (21) can be converted using the following averaging operator:

$L\left[\varphi_{k}\left(x_{i}, t\right)\right]=V_{k}^{-1} \cdot \int_{V_{k}} \varphi_{k}\left(x_{i}, t\right) d V_{k}=\varphi_{k} V(t) ;$

considering that the initial temperature $T_{k v}(0)=T_{k}^{b}$;

$\lambda_{k}=\left(c_{\rho}\right)_{k} \rho_{k} a_{k} ; C_{k}=\left(c_{\rho}\right)_{k} \rho_{k} V_{k}$ - full heat capacity $\left.[\mathrm{J} / \mathrm{K}]\right)$, will result in

$C_{k} \frac{\partial T_{k v}(t)}{\partial t}+\psi_{\alpha k} T_{\alpha k}+\sum_{\substack{l=1 \\ k \neq l}}^{n} \psi_{k l} \cdot\left[\left(T_{k}\right)_{A_{l}}-\left(T_{l}\right)_{A_{k}}\right]=Q_{e k}$

Expression (23) can also be recorded in a matrix form:

$[\boldsymbol{C}] \times \frac{\partial[\boldsymbol{T}]}{\partial t}+[\boldsymbol{\alpha}] \times[\boldsymbol{T}]=[\boldsymbol{Q}] ;$

For different types of thermal links between bodies and those between bodies and the environment, the thermal resistance $\mathrm{R}$ will be determined by the following equations:

$R=\frac{1}{A \alpha} ;\left[\frac{K}{W}\right]$;

- during thermal coupling, when heat is transferred by convection at the border between the body and liquid (gas);

$R=\sum_{i=1}^{n} R_{i}=\frac{1}{A} \cdot \sum_{i=1}^{n} \frac{h_{i}}{\lambda_{i}} ;\left[\frac{K}{W}\right]$

- during thermal coupling, when heat is transferred through a composite flat wall of consecutive bodies;

$R=\sum_{i=1}^{n} R_{i}=\frac{1}{A} \cdot \sum_{i=1}^{n}\left(\frac{1}{\alpha_{1}}+\frac{h_{i}}{\lambda_{i}}+\frac{1}{\alpha_{2}}\right) ;\left[\frac{K}{W}\right]$

- in case of thermal coupling, when heat is transferred through a composite flat wall of consecutive bodies at the borders of which there is a heat exchange with liquid or gas with heat transfer coefficients equal to $\alpha_{1}$ and $\alpha_{2}$ respectively;

$R=\sum_{i=1}^{n} R_{i}=\frac{1}{A_{1}} \cdot \sum_{i=1}^{n}\left(\frac{1}{\alpha_{1}}+\frac{h_{i}}{\lambda_{i}}+\frac{A_{1}}{A_{2} \cdot \alpha_{2}}\right) ;$

- in thermal coupling, when heat is transferred through a composite flat wall of sequential bodies of different areas $\mathrm{A}_{1}$ and $A_{2}$, at the borders of which there is a heat exchange with liquid or gas, with heat transfer coefficients equal to $\alpha_{1}$ and $\alpha_{2}$ respectively;

$R=\sum_{i=1}^{n} R_{i}=\frac{1}{L d_{1} \alpha_{1}}+\sum_{i=1}^{n} \frac{1}{2 \pi L \lambda_{i}} \cdot \ln \left(\frac{d_{i+1}}{d_{i}}\right)+\frac{1}{L d_{i+1} \alpha_{2}} ;\left[\frac{K}{W}\right]$

- in case of thermal coupling, when heat is transferred through a cylindrical wall or a cylindrical wall of sequential bodies at the borders of which there is a heat exchange with liquid or gas with heat transfer coefficients equal to $\alpha_{1}$ and $\alpha_{2}$ respectively;

$R^{-1}=\sum_{i=1}^{m} \frac{1}{R_{i}} ;\left[\frac{W}{K}\right]$

- in thermal coupling, when the heat is transferred by a set of different methods that are not dependent on each other.

$T_{j}=T_{j}^{b}+\left[\frac{Q_{e q}+Q_{j} \cdot\left(1+\frac{\psi_{e q \alpha}}{\psi_{j e q}}\right)}{\psi_{\alpha j}+\psi_{e q \alpha} \cdot\left(1+\frac{\psi_{\alpha j}}{\psi_{j e q}}\right)}-T_{j}^{b}\right]$.
$\left[\frac{m_{2 j} \cdot\left(1-e^{-m_{1 j} t}\right)-m_{1 j} \times\left(1-e^{-m_{2 j} t}\right)}{m_{2 j}-m_{1 j}}\right]+\left[\frac{Q_{j}-T_{j}^{b} \psi_{\alpha j}}{C_{j} \cdot\left(m_{2 j}-m_{1 j}\right)}\right] \cdot[(1-$
$\left.\left.e^{-m_{2 j} t}\right)-\left(1-e^{-m_{1 j} t}\right)\right] ;$

In most standard machine tools, when for the $j$-component of the machine tool, the thermal fluxes are constant 
$Q_{j}=$ const solving equation (23) temperature $T_{j}$ obtains the $j$ - component following (31), where

$$
\begin{aligned}
& Q_{e q}=\sum_{\substack{k=1 \\
k \neq j}}^{n} Q_{i k} ; \psi_{j e q}=\sum_{\substack{k=1 \\
k \neq j}}^{n} \psi_{j k} ; \psi_{e q \alpha}=\sum_{\substack{k=1 \\
k \neq j}}^{n} \psi_{\alpha k} ; \\
& C_{e q}=\sum_{\substack{k=1 \\
k \neq j}}^{n} C_{k} ; T_{e q}^{b}=\sum_{\substack{k=1 \\
k \neq j}}^{n} \frac{\psi_{j k}}{\psi_{j e q}} \cdot T_{k}^{b} ; m_{1,2}=\frac{1}{2} \cdot\left(H_{1 j} \pm\right. \\
& \left.\sqrt{H_{1 j}^{2}-4 H_{2 j}}\right) ;[1 / \mathrm{h}] ; H_{1 j}=\frac{\psi_{j e q}+\psi_{\alpha j}}{C_{j}+\frac{\psi_{j e q}+\psi_{e q \alpha}}{C_{e q}}} ;[1 / \mathrm{h}] ; \\
& H_{2 j}=\frac{\psi_{j e q}}{C_{j} \cdot C_{e q}} \cdot\left(\psi_{\alpha j}+\psi_{e q \alpha}+\frac{\psi_{\alpha j} \psi_{e q \alpha}}{\psi_{j e q}}\right) ;\left[1 / h^{2}\right] .
\end{aligned}
$$

Fig. 5 shows typical normalized regularities of the change in time of the machine parts' temperature, which are obtained by dependence (23), based on the range and mutual ratio of growth rate and based on temperature values in the established mode for machine tools [7].

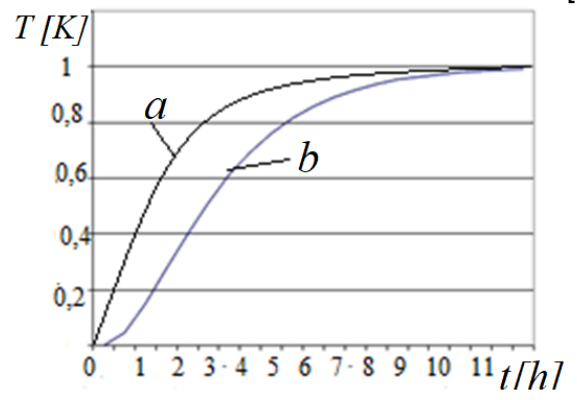

Fig. 5 Character of typical normalized change in time temperature of machine tool parts.

It is evident that the typical pattern given in Fig. 5 a is typical for all cases of heating - cooling of parts and is a "quasiexponential" dependence. At the initial stage of heating, the function has an explicit concave nature with the point of inflection after some time. The exponential character of change in time, Fig. 5 b, regarding temperature of a detail occurs only at equality in expression (23) growth rates.

$m_{j}=m_{1 j}=m_{2 j}=\frac{\psi_{\alpha j}+\psi_{e q \alpha}}{C_{j}+C_{e q}} ;$

and at $\psi_{j e q} \rightarrow \infty$, when the $\psi_{k l}$ thermal conductance of the joints of the parts is quite high:

$T_{j}=T_{j}^{b}+\left(\frac{Q_{e q}+Q_{j}}{\psi_{\alpha j}+\psi_{e q \alpha}}-T_{j}^{b}\right) \cdot\left(1-e^{-m_{j} t}\right) ;$

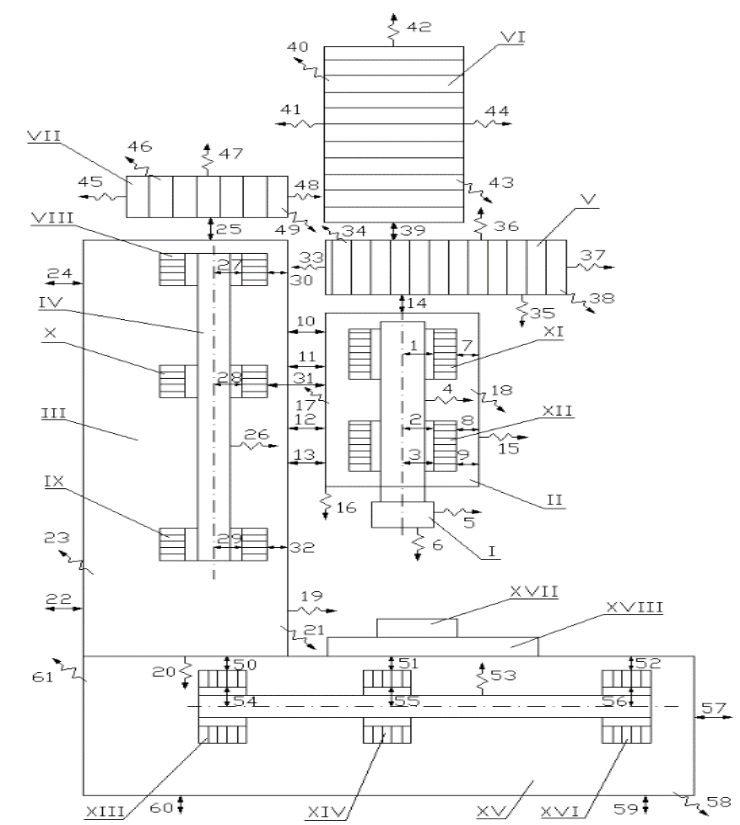

Fig. 6 Temperature model of vertical milling machine with heat-active elements I-XVIII and thermal links 1-60.
Fig. 6 shows the diagram of temperature analysis for a vertical milling machine with indicated heat-active elements from I to XVIII and thermostable links.

The solution of equation (23), depending on the requirements, can be obtained for different conditions of heat links accounting. The obtained temperature values are the basis for the solutions to determine the displacements in equations (7) and other related problems.

\section{THERMAL STIFFNESS AS A KEY ACCURACY INDICATOR}

Equation (7) is a mathematical expression of a generalized thermophysical structure, describing an arbitrary thermophysical structure of a metal cutting machine consisting of $n$ thermostable links built on $i=n-1$ heatactive elements. Based on equation (7) and (23) and on the analysis of variation ranges of geometrical and thermophysical parameters of machine tools, Fig. 7 shows graphs of varying normalized values of average nonstationary temperatures $T^{0}(t)$ and thermoelastic deformations $\delta r(t)$ of machine tools.

The graphs in Fig. 7 show different machine responses in the form of temperature variations and temperature deformations. The machine tools' properties to resist (temperature stiffness) thermal influences are different, therefore, they should be described by different parameters of temperature stiffness estimation.
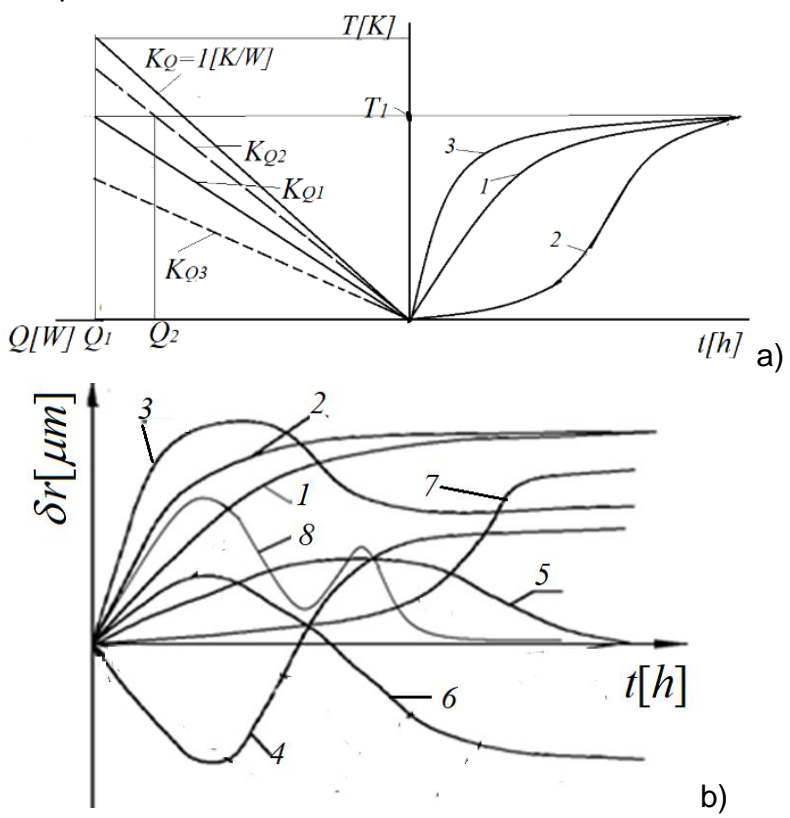

b)

Fig. 7 Typical normalized variations a) in average temperatures ( 1 - 3 types) $T^{0}(t)$ and b) thermoelastic deformations $\delta r(\mathrm{t})$ (types 1-8) of machine tools.

The indices of the machine's general temperature and thermoelastic characteristics are to be defined, which will allow an estimation and change of the machine tool's property to resist the thermal influences with the given sensitivity and accuracy. For simplicity, only the first two terms of the vector $\delta r=\left|\delta r_{x} \delta r_{y} \delta r_{z} 1\right|^{T}$ of equation (7) are considered, and its projection on the coordinate axis. For example, a projection on the $O Y$ axis will result in the following:

$\delta r_{y}=K_{1} M_{1 y} \delta r_{1 y}^{0}+K_{2} \delta r_{2 y}^{0}+M_{1 y}\left(\boldsymbol{B}_{1 y}^{\alpha}\right)^{I} r_{1 y}+\left(\boldsymbol{B}_{2 y}^{\alpha}\right)^{I} r_{2 y}+$ $K_{1} M_{1 y}\left(\boldsymbol{B}_{1 y}^{\alpha}\right)^{I} \delta r_{1 y}^{0}+K_{2}\left(\boldsymbol{B}_{2 y}^{\alpha}\right)^{I} \delta r_{2 y}^{0}=\left[\left(M_{1 y}+\right.\right.$

$\left.M_{1 y}\left(\boldsymbol{B}_{1 y}^{\alpha}\right)^{I}\right] K_{1} \delta r_{1 y}^{0}+\left(1+\left(\boldsymbol{B}_{2 y}^{\alpha}\right)^{I}\right) K_{2} \delta r_{2 y}^{0}+M_{1 y}\left(\boldsymbol{B}_{1 y}^{\alpha}\right)^{I} r_{1 y}+$ $\left(\boldsymbol{B}_{2 y}^{\alpha}\right)^{I} r_{2 y}=\left[\left(M_{1 y}+M_{1 y}\left(\boldsymbol{B}_{1 y}^{\alpha}\right)^{I}\right] \cdot K_{1} \delta r_{1 y}^{0}+\left(1+\left(\boldsymbol{B}_{2 y}^{\alpha}\right)^{I}\right)\right.$. 
$K_{2} \delta r_{2 y}^{0}+M_{1 y}\left(\boldsymbol{B}_{1 y}^{\alpha}\right)^{I} r_{1 y}+\left(\boldsymbol{B}_{2 y}^{\alpha}\right)^{I} r_{2 y}=\left\{\left[\left(M_{1 y}+\right.\right.\right.$

$\left.M_{1 y}\left(\boldsymbol{B}_{1 y}^{\alpha}\right)^{I}\right]+\left(1+\left(\boldsymbol{B}_{2 y}^{\alpha}\right)^{I}\right) \cdot \frac{K_{2}}{K_{1}} \cdot \frac{T_{2} r_{2 y}}{T_{1} r_{1 y}}+M_{1 y}\left(\boldsymbol{B}_{1 y}^{\alpha}\right)^{I} \cdot \frac{1}{K_{1} \beta T_{1}}+$

$\left.\left(\boldsymbol{B}_{2 y}^{\alpha}\right)^{I} \cdot \frac{r_{2 y}}{r_{1 y}} \cdot \frac{1}{K_{1} \beta T_{1}}\right\} \cdot K_{1} \beta r_{1 y} T_{1}$;

where $\quad M_{1 y}=(-\sin x \sin y \cos z+\cos x \sin z) \cdot \frac{\delta r_{1 x}^{0}}{\delta r_{1 y}^{0}}+$ $\sin x \sin y \sin z+(\cos x \cos z-\sin x \cos y) \cdot \frac{\delta r_{1 z}^{0}}{\delta r_{1 y}^{0}} ;\left(\boldsymbol{B}_{1,2 y}^{\alpha}\right)^{I}=$ $\left[-\delta \alpha_{x} \delta \alpha_{y} \cdot\left(1-\frac{\delta \alpha_{z}^{2}}{2}\right)+\left(1-\frac{\delta \alpha_{x}^{2}}{2}\right)\right] \cdot \delta \alpha_{z} \frac{r_{1,2 x}}{r_{1,2 y}}-$ $\delta \alpha_{z} \delta \alpha_{x} \delta \alpha_{y}+\left[\left(1-\frac{\delta \alpha_{y}^{2}}{2}\right) \cdot\left(1-\frac{\delta \alpha_{z}^{2}}{2}\right)-\delta \alpha_{x}\left(1-\frac{\delta \alpha_{y}^{2}}{2}\right)\right]$. $\frac{r_{1,2 z}}{r_{1,2 y}} ; \alpha_{e}=\frac{M_{T}}{E J}+C_{1}=\beta K_{c} \zeta T ; e=x, y, z ; \zeta_{i},[0-1]-$ the coefficient of non-uniformity of temperature distribution of a heat-active element characterizes the non-uniformity of the temperature field in the cross-section, and if the temperature gradients in the directions and cross-section are close or equal, the values of $\zeta_{i} \rightarrow 1, K_{c}$ - geometrical form factor in links is equal to $\frac{r_{z}^{2}}{8 r_{y}}$ for a free beam, $\frac{r_{z}^{2}}{2 r_{y}}$ for a one-side fixed beam.

Analyzing the components of the expression (34), it is concluded that the matrix components are not thermophysical or mass-dimensional parameters and therefore, do not affect the thermal characteristics of the machine. Thus, in future analysis, this expression will be simplified by considering them as single matrices, and the product of the components $\left(\boldsymbol{B}^{\alpha}\right)^{I} \delta r^{0}$ are the values of 2, 3, 4 orders of magnitude relatively. In addition, $\delta r$ has an insignificant effect on both the size and the temperature characteristics of the machine. Then the expression (34) will be simplified and documented.

$\delta r_{y}=K_{1} M_{1 y} \delta r_{1 y}^{0}+K_{2} \delta r_{2 y}^{0}+M_{1 y}\left(\boldsymbol{B}_{1 y}^{\alpha}\right)^{I} r_{1 y}+\left(\boldsymbol{B}_{2 y}^{\alpha}\right)^{I} r_{2 y}=$ $K_{1} \delta r_{1 y}^{0}+K_{2} \delta r_{2 y}^{0}+\left(\boldsymbol{B}_{1 y}^{\alpha}\right)^{I} r_{1 y}+\left(\boldsymbol{B}_{2 y}^{\alpha}\right)^{I} r_{2 y}=K_{1} \beta T_{1} r_{1 y}+$ $K_{2} \beta T_{2} r_{2 y}+\left(\delta \alpha_{1 z}-\delta \alpha_{1 x}\right) \cdot r_{1 y}+\left(\delta \alpha_{2 z}-\delta \alpha_{2 x}\right) \cdot r_{2 y}=$ $K_{1} \beta T_{1} r_{1 y}+K_{2} \beta \zeta_{1 y} T_{1} r_{2 y}+\left(\beta K_{c} \zeta_{1 z} T_{1}-\beta K_{c} \zeta_{1 x} T_{1}\right) \cdot r_{1 y}+$ $\left(\beta K_{c} \zeta_{2 z} \zeta_{1 y} T_{1}-\beta K_{c} \zeta_{2 x} \zeta_{1 y} T_{1}\right) \cdot r_{2 y}=\left[K_{1}+K_{2} \zeta_{1 y} \cdot \frac{r_{2 y}}{r_{1 y}}+\right.$ $\left.\left(K_{1 c} \zeta_{1 z}-K_{1 c} \zeta_{1 x}\right)+\left(K_{2 c} \zeta_{2 z} \zeta_{1 y}-K_{2 c} \zeta_{2 x} \zeta_{1 y}\right) \cdot \frac{r_{2 y}}{r_{1 y}}\right]$.

$\beta T_{1} r_{1 y} K_{\beta j} T_{1} r_{1 y}=K_{\lambda j} \cdot T_{1}=K_{T j}^{-1} \cdot T_{1} ;$

where $K_{i}$ - relative stiffness coefficient of a heat-active element at thermostable links;

$K_{\beta j}=\beta \cdot\left[K_{1}+K_{2} \zeta_{1 y} \cdot \frac{r_{2 y}}{r_{1 y}}+\left(K_{1 c} \zeta_{1 z}-K_{1 c} \zeta_{1 x}\right)+\right.$

$\left.\left(K_{2 c} \zeta_{2 z} \zeta_{1 y}-K_{2 c} \zeta_{2 x} \zeta_{1 y}\right) \cdot \frac{r_{2 y}}{r_{1 y}}\right]$ - the given coefficient of temperature deformations of the machine $[1 / \mathrm{K}] ; K_{\lambda j}=$ $K_{\beta j} r_{1 y}$ - temperature elasticity coefficient $[\mu \mathrm{m} / \mathrm{K}] ; K_{T j}=$ $\frac{1}{K_{\beta j} r_{1 y}}=\frac{1}{K_{\lambda j}}$ - temperature stiffness $[\mathrm{K} / \mu \mathrm{m}]$.

Substituting the value of temperature (31) in expression (35) will result in the value of the machine's thermoelastic deformation reduced to the first heat-active element, i.e., to the spindle of the machine at the TCP and at the time value $t \rightarrow \infty$, equation (34) will be changed to:

$$
\begin{aligned}
& \delta r_{y}=K_{T j} \cdot T_{1}=K_{T j} \cdot \frac{Q_{e q}+Q_{j}\left(1+\frac{\psi_{e q \alpha}}{\psi_{j e q}}\right)}{\psi_{\alpha j}+\psi_{e q \alpha}\left(1+\frac{\psi_{\alpha j}}{\psi_{j e q}}\right)}=K_{T j} . \\
& \frac{\left(\frac{Q_{e q}}{Q_{j}}\right)+\left(1+\frac{\psi_{e q \alpha}}{\psi_{j e q}}\right)}{\psi_{\alpha j}+\psi_{e q \alpha}\left(1+\frac{\psi_{\alpha j}}{\psi_{j e q}}\right)} \cdot Q_{j}=K_{\lambda j} \cdot \frac{\theta_{Q}+\left(1+\frac{\psi_{e q \alpha}}{\psi_{j e q}}\right)}{\psi_{\alpha j}+\psi_{e q \alpha}\left(1+\psi_{\alpha j} / \psi_{j e q}\right)} \cdot Q_{j}= \\
& K_{\lambda j} \cdot K_{\alpha j} \cdot Q_{j}=K_{Q j}^{-1} \cdot Q_{j}
\end{aligned}
$$

where $\theta_{Q}=\frac{Q_{e q}}{Q_{j}}$ - the ratio of the machine's internal heat sources to the source of the first heat-active element; $K_{\alpha j}=\frac{\theta_{Q}+\left(1+\frac{\psi_{e q \alpha}}{\psi_{j e q}}\right)}{\psi_{\alpha j}+\psi_{e q \alpha}\left(1+\frac{\psi_{e q \alpha}}{\psi_{j e q}}\right)}-$ heat stiffness $[\mathrm{K} \mathrm{W}] ; K_{\theta j}=\frac{1}{K_{\alpha j}}$ - heat elasticity [W/K]; $K_{Q j}=K_{T j} \cdot K_{\theta j}=\frac{1}{K_{\lambda j}} \cdot \frac{1}{K_{\alpha j}}-$

thermal stiffness $[\mathrm{W} / \mu \mathrm{m}]$

Heat, temperature, and thermal stiffness values of the machine are determined in the same way in other axial directions of $\mathrm{OX}$ and $\mathrm{OZ}$ since obviously, these stiffness values are different due to the thermophysical uniqueness of machine design and layout solutions.

\section{SUMMARY}

Heat-active elements form a thermophysical structure with the support of quasi-thermostable links in a kinematic chain. Thermophysical equations describe the heat flow by laws of conduction and convection and result in temperature changes. Heat-active elements change the linear sizes by thermoelastic deformations, and the inhomogeneity of temperature fields causes their angular rotation (bending). Heat stiffness, temperature stiffness, and thermal stiffness values of the machine are determined by the thermoelastic deformation $\delta r$ between the TCP and the workpiece coordinate system, mechanical stiffness, preload, clearance, and non-uniformity in the coefficients of temperature. Examples of the thermal stiffness are given for several machine tool types.

\section{REFERENCES}

[Dm 2018] Dmitriev B. M. Assessing the Thermal Rigidity of a Metal-Cutting Machine. Russian Engineering Research. Vol. 38, No. 2, 2018, pp.94-98.

[Gro 2015] Großmann Knut. Thermo-energetic Design of Machine Tools. Springer International Publishing Switzerland, 2015, 261p.

[ISO 2007] ISO230-3:2007, Test code for machine tools. Part 3: Determination of thermal effects.

[Kuz 2011] Кузнецов А.П. Тепловое поведение и точность металлорежущих станков. - М.: Янус-К, 2011. 256 c. Kuznetsov, A.P., Teplovoe povedenie i tochnost' metallorezhuchchikh stankov, M.: Yanus-K, 2011, 256p.

[Kuz 2018] Kuznetsov A.P. and Koriath H.-J.: The Methods for Controlled Thermal Deformations in Machine Tools. ICTIMT, Verlag Wissenschaftliche Scripten, Dresden, 2018, p. 47-60.

[Kuz 2019] Кузнецов А.П.: Тепловые процессы в металлорежущих станках. - М.: Техносферера, 2019. 488c. (Kuznetsov A. P.: Thermal processes in machine tools. - M.: Technosphere, 2019, 488p.)

[May 2012] Mayr J. and Jedrzejewski J.: Thermal issues in machine tools. CIRP Annals - Manufacturing Technology, 2012, (61) Switzerland, 2015, 261p., pp. 771-791.

[Mek 2009] Mekid S. Introduction to precision machine design and error assessment. CRC Press, 2009, 330p.

[Sok 1968] Соколов Ю.Н. Температурные расчеты в станкостроении. - М.: Машиностроение, 1968, 77с. Sokolov Y.N.: Temperaturnye razshety $v$ stankostroyeniyMoscow, Mashinostroyenie, 1968, 77p.

[Yto 2010] Yto Y.: Thermal deformation in Machine tools. McGraw-Hill, 2010, 214p 